Research Article

\title{
Failure Mechanism of Rock Slopes under Different Seismic Excitation
}

\author{
Hua Tang $\mathbb{D}^{1}{ }^{1}$ Zhenjun Wu $\mathbb{D},{ }^{1}$ Ailan Che $\mathbb{D}^{2},{ }^{2}$ Conghua Yuan, ${ }^{1}$ and Qin Deng ${ }^{1}$ \\ ${ }^{1}$ State Key Laboratory of Geomechanics and Geotechnical Engineering, Institute of Rock and Soil Mechanics, \\ Chinese Academy of Sciences, Wuhan, China \\ ${ }^{2}$ School of Naval Architecture, Ocean and Civil Engineering, Shanghai Jiao Tong University, Shanghai, China
}

Correspondence should be addressed to Hua Tang; htang@whrsm.ac.cn

Received 28 September 2020; Revised 25 January 2021; Accepted 9 February 2021; Published 20 February 2021

Academic Editor: Guoqiang Xie

Copyright (C) 2021 Hua Tang et al. This is an open access article distributed under the Creative Commons Attribution License, which permits unrestricted use, distribution, and reproduction in any medium, provided the original work is properly cited.

In earthquake-prone areas, special attention should be paid to the study of the seismic stability of rock slope. Particularly, it becomes much more complicated for the rock slopes with weak structural surfaces. In this study, numerical simulation and the shaking table test are carried out to analyze the influence of seismic excitation and structural surface in different directions on dynamic response of rock slope. Huaping slope with bedding structural surfaces and Lijiang slope with discontinuous structural surfaces besides Jinsha River in Yunnan Province are taken as research objects. The results of numerical simulation and the model test both show that discontinuous structure surface has influence on the propagation characteristics of seismic wavefield. For Huaping slope, the seismic wavefield responses repeatedly between the bedding structural surface and slope surface lead to the increase of the amplification effect. The maximum value of seismic acceleration appears on the empty surface where terrain changes. Horizontal motion plays a leading role in slope failure, and the amplification coefficient of horizontal seismic acceleration is about twice that of vertical seismic acceleration. The failure mode is integral sliding along the bedding structural surface. For Lijiang slope, seismic acceleration field affected by complex structural surface is superimposed repeatedly in local area. The maximum value of seismic acceleration appears in the local area near slope surface. And the dynamic response of slope is controlled by vertical and horizontal motion together. Under the seismic excitation with an intense of $0.336 \mathrm{~g}$ in $X$ direction and $Z$ direction, the amplification coefficients of seismic acceleration of Lijiang slope are 3.23 and 3.18, respectively. The vertical motion leads to the cracking of the weak structural surface. Then, Lijiang slope shows the toppling failure mode under the action of horizontal motion.

\section{Introduction}

It is widely reported that a lot of earth embankments or slopes were damaged during the past great earthquakes [1-4]. Numerous substantial landslides have been triggered by earthquakes and caused severe loss of property and life [5-8]. On 12 May 2008, the Wenchuan earthquake occurred in the Longmenshan tectonic zone in Sichuan Province, China, which epicenter was located at the eastern edge of Qinghai-Tibet Plateau [9, 10]. This earthquake not only caused extensive damage to rural houses, educational and healthcare facilities, and lifeline engineering systems but also induced an abundance of landslides and other geologic disasters, such as collapses, debris flow, and shattered mountains [11]. It can be found that the seismic landslides hazards have been the hot topic in geotechnical and earthquake engineering [12-14].

Among these earthquake-induced landslides events, the failure of the bedding slopes, which represents slopes with weak structural plane in the slope, was particularly notable because of sudden and rapid sliding [15]. Due to the complexities of the slope compositions, rock mass discontinuities, and the nature of earthquake-induced ground motion, it is difficult to quantitatively assess failure potentials $[8,16,17]$. The mechanical properties of the rock mass are deteriorated by the weak intercalated layers, extension, and convergence of the joints in the layered rock mass [17-19]. The dynamic response characteristics and failure 
mode of the slopes with weak structural plane should be further studied. In particular, the influence of weak structural plane on the wave propagation in the rock slopes with discontinuities should be analyzed in detail. To mitigate the influence of landslide hazards, it is necessary to understand the deformation behavior of slopes with structural planes under seismic excitation.

Many scholars have performed a lot of research on dynamic response of rock slope [12, 20-22]. It is worth noting that the direction of seismic wave propagation has a great influence on the dynamic response of the rock slope $[7,8,12]$. In the field surveys of geological disasters after the earthquake, some large rocks can be found to be thrown horizontally, such as in the Wenchuan earthquake, which is closely related to the direction of seismic waves $[11,23,24]$. In general, rock masses on slopes are collapsed by P-waves and S-waves. P-waves mainly induce many lateral cracks on the slope surface, while $S$-waves also trigger many fractures inside the slope. Under the combined of the two effects, landslides and collapses will occur eventually $[7,8,12]$. Most of the landslides near the epicenter of the Wenchuan earthquake were subjected to strong lateral $\mathrm{S}$-wave forces and were characterized by "throw-like collapses." In addition, [25, 26] studied the dynamic response characteristics of the slope with a weak interlayer under the action of seismic forces in different directions. Song et al. [17] studied the influence of the input directions of different seismic waves on the dynamic response of a rock slope with discontinuous joints by using the shaking table test. Fan et al. [19] explored the seismic stability of a bedding slope under the action of different seismic load directions. The above conclusions indicate that the input direction of ground motion has great influence on the dynamic response characteristics of the rock slope. Given the abovementioned analysis, the dynamic stability of rock slope with complex geological structure under different directions of ground motion needs further research.

Earthquakes are caused by a large amount of energy released from the Earth's interior as the plates and shells squeeze on the fault surface. Ground motion is divided into near-field motion and far-field motion. In particular, the damage to buildings is more intense in the near site vibration, and the damage to buildings is less in the far site vibration due to the energy dissipation caused by the longdistance transmission of seismic waves [27-29]. After the near-field earthquake, the surrounding rock and soil mass produced large ground vibrations, especially the P-wave reached the ground quickly, which is also the reason why the ground rock and soil mass first produced vertical ground motion. After the far-field seismic ground motion, due to the long-distance transmission, the longitudinal wave component of the seismic wave gradually weakens, and the influence on the far-field is mainly carried out by shear wave $[30,32]$. It can be seen that compared with far-field wave, the effect of longitudinal wave is more serious under the action of near-field vibration. In order to further study this phenomenon, numerical calculation and the shaking table test are carried out as follows.
The model test is an effective method to investigate the dynamin response of rock-soil mass with nonlinear stress behavior. In recent years, the shaking table tests have been widely used to study the seismic response of various types of lithology combination and structures [12, 20, 33]. The shaking table test method has been one way to simulate the variation of dynamic response of slope and directly reveal the failure modes of structure under seismic excitations $[19,22]$. The problem of wave propagation in discontinuous media has been studied by many researchers $[21,25,26,34-36]$, but the problem associated with rock slope comprising weak intercalated layers has not been widely discussed. Except the shaking table test, the centrifuge test is also a common method to study the failure process of rock slope under seismic motion. A geotechnical centrifuge could offer the same stress and strain level within the scaled model by testing at $N$ times Earth's gravity [37, 38]. Compared to the $1 \mathrm{~g}$ shaking table test, the centrifuge test has the better ability to model the linear dimension of gravitational acceleration based on the centrifugal force $[39,40]$. However, the scale of the centrifuge test is generally small due to the size limitation of equipment, restraining the spatial dynamic response analysis of the slope model.

Wave propagation in the rock slopes with discontinuity joints, and the influence of these heterogeneities on slope stability has become topics of interest in rock dynamics and earthquake engineering. In order to evaluate the effects of the discontinuity joints on the dynamic response in rock mass slopes, two-dimensional finite element models with steep-inclined structural planes and weak intercalated layers are developed for the dynamic analysis. Moreover, a series of large-scale shaking table tests are conducted to investigate the effects of steep-inclined structural planes and weak intercalated layers on the wave propagation in rock slopes and to evaluate the influence of wave propagation on the slope dynamic stability. The comparison of the dynamic response obtained from the model test and numerical simulation and the failure mechanism are well discussed.

\section{Case Study}

The study area is in the transition zone between the southeast edge of the Tibetan Plateau and the Yunnan-Kweichow Plateau in Yunnan Province, Southwest China. Figure 1 shows the location of the study area. The neotectonic movement is strong in the study area, and several faults pass through the area. In the recent 10 years, about 20 earthquakes occur in Yunnan Province, for example, on August 28 and 31, 2013, two earthquakes with Ms5.1 and Ms5.9 occurred on the boundary between Deqing County, Yunnan Province, and Derong County, Sichuan Province; moreover, on $3^{\text {rd }}$ August, 2014, the Ludian Ms6.5 earthquake sequence occurred in Yunnan Province.

As the key project of Huali highway, Jinsha River Bridge is located in the tributary of Jinsha River. The bank slope has stepped topography due to the dip structure and flow along the river. Huaping slope is along the river, with the gradient of $20^{\circ}-30^{\circ}$. The exposed strata of project area are mainly Permian Basalt formation (P2 $\beta 3$ ), Xuan Wuyan, Xuan 
Wuyan almond-shaped lithologic volcano, breccia lava, and tuff. The pile foundation of the main pier is embedded in the zone between tuff structure surface and steep structural surface in the trailing edge (Figure 2(a)). The simplified geological profile of the slope is shown in Figure 3(a). The physical and mechanical parameters of rock material from the direct shear test and field test are listed in Table 1. The Lijiang bank slope is located on the right side of Jinsha River against Huaping slope, and the average natural gradient of the slope is about $40^{\circ}$ (Figure 2(b)). The thickness of soil is $0-5 \mathrm{~m}$ on the slope surface, including colluvial rock mass and brecciated soil. Many joints and fissures develop in rock masses, and the mountain unloading effect is strong. The simplified geological profile of the slope is shown in Figure 3(b).

\section{Numerical Simulation}

FEM dynamic analyses were performed to clarify the characteristics of seismic wave propagation in rock mass with joints. The research object is simplified as plane strain problem, and the size, degree of subdivision, and boundary conditions of the FEM models have been optimized to effectively simulate wave propagations in a discontinuous medium [26].

3.1. Numerical Model. To clarify the effects of the discontinuous joints on the dynamic response of the slopes, 2 types of slopes were modeled, which are case 1: Huaping slope, case 2: Lijiang slope, as shown in Figure 4. The dimensions and details of the meshed model are illustrated in Figure 4. The Huaping slope size is $431,000 \times 900,000 \mathrm{~mm}$ with its slope gradient about approximately $20-30^{\circ}$, and the discontinuity joints were set $1 \mathrm{~m}$ in thickness. The Lijiang slope size is $829,000 \times 390,000 \mathrm{~mm}$ with its slope gradient about $40^{\circ}$. The weak structural surfaces were set $1 \mathrm{~m}$ in thickness, with a $15^{\circ}$ (bedding) and $165^{\circ}$ (toppling) gradients in the rock slope, as shown in Figure 4(b).

This study mainly considers the impact of the physical mechanical property of discontinuous joints on the wave propagation characteristics in rock mass. Therefore, the tie connection method between the discontinuous joints and rock mass was adopted, rather than the surface connection method without setting viscous damping. The total number of 109284 plane strain elements was generated in the finite element model. The assumed mechanical properties of the rock mass are listed in Table 1; the materials are considered as elastic. Infinite elements were set around the model to reduce the boundary effect caused by seismic wave reflection.

The input motion was synthesized taking into account the engineering geological conditions and historical seismic data at the bank slope, based on the safety evaluation of the ground motion parameters of the Jinsha River Bridge on the Huali Expressway. The synthetic seismic waveform was applied at the bottom boundary of the model with a frequency of $f=2-7 \mathrm{~Hz}, t=0.05 \mathrm{sec}$, and $\Delta t=0.005 \mathrm{sec}$ to create time-dependent acceleration inputs at the bottom of the slope during horizontal and vertical motions in the calculations, as shown in Figure 5.

3.2. Analysis Results. The numerical results of the Huaping and Lijiang slope are shown in Figures 6 and 7, respectively. The initial stress resulting from gravity was not considered.

Figure 6 shows the distribution of the maximum acceleration response for the Huaping slope under horizontal and vertical seismic loads. It can be seen that the acceleration response of Huaping slope increases gradually along the height direction in mutant at the structural plane. The acceleration change of the slope below the structure surface is not obvious. The acceleration amplification has a sudden increase when it passes through the structural plane and reaches its maximum value ( 4.3 times) at the top of the trailing edge and the middle of the slope surface, respectively. The influence of the bedding structural surface on the rock slope is significant. It can be summarized that the seismic wave propagates upward along the slope surface. The structural surface and slope surface will refract and reflect the seismic wave. The superposition of the reflected and refracted seismic wave and the seismic wave from the slope toe causes the acceleration response to increase when the position of the slope surface changes, such as the empty surface. So, the amplification effect of acceleration is more predominant at the top of slope and the change of slope surface, as shown in Figure 6. The trailing edge of steep structure surface alters the peak acceleration around the region, which can lead to shear failure of the tuff on the structure surface and consequently reduce the dynamic stability of rock slope. Moreover, it can be found that compared with the vertical seismic motion, slope with structure surface conditions has a more obvious amplification effect on the horizontal ground motion, and the seismic wave directions have a great impact on the dynamic response of the slope.

To clarify the acceleration amplification effect of the Lijiang slope with discontinuous structural planes, the acceleration distributions when input in $x$ and $z$ directions are shown in Figure 7. Figure 7 shows that the PGA is much larger in the surface slope above the topmost bedding structural plane, indicating that the surface slope is the most unstable area during earthquakes. In particular, the PGA near the platform is the largest, which shows that the platform area will be initially damaged under earthquakes. The distribution of the acceleration maximum shows irregular characteristics. As a whole, the absorption of seismic energy phenomena can be identified inside the slope in Figure 7, but the local amplification effect can be also found between the bedding structural surfaces. Because of the existence of complex discontinuous structural surface, the slope is divided into many blocks. The reflection and refraction of seismic wave in blocks are more complex. The seismic wave reflected and refracted in a block is superposed with the seismic wave from the slope toe, so that the acceleration reaches the maximum value. In addition, Figure 7 shows that the seismic wave direction has a great impact on 


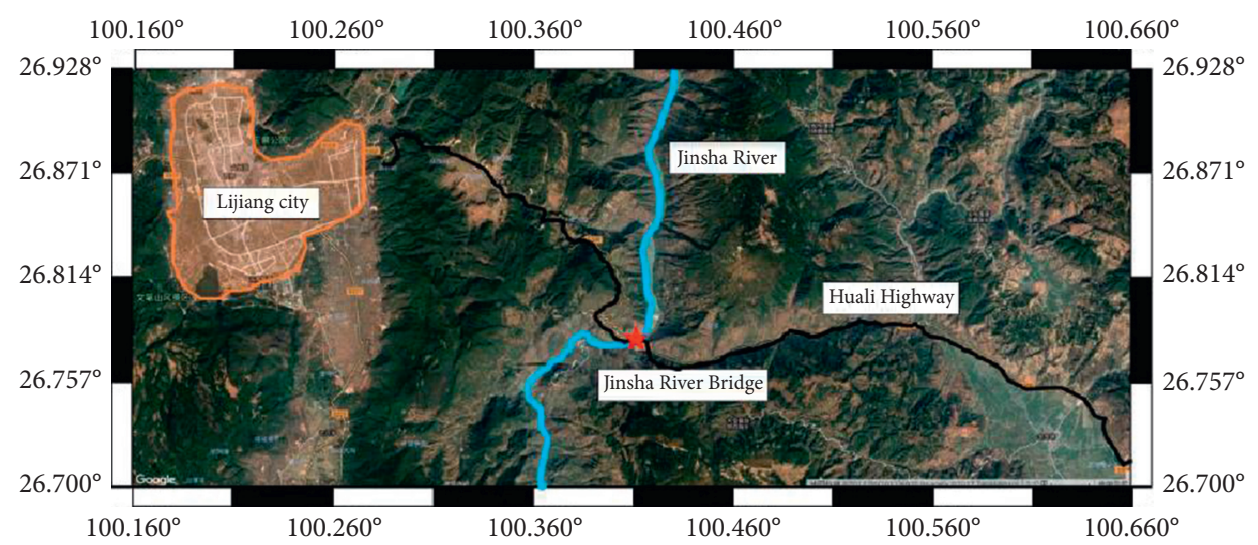

Figure 1: Location of the study area.

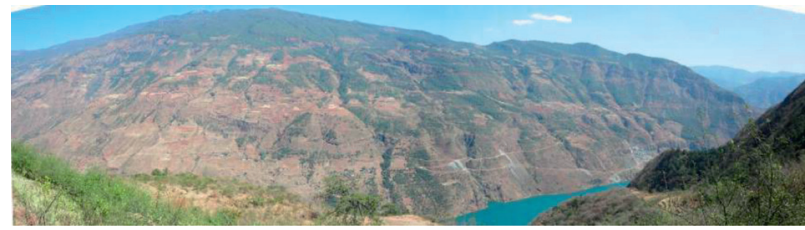

(a)

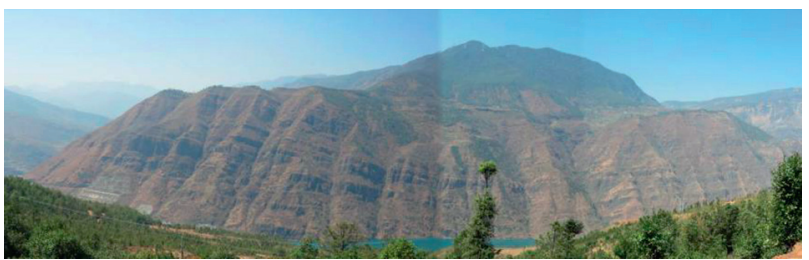

(b)

FIgURE 2: Topography and geomorphology of the slopes: (a) Huaping slope; (b) Lijiang slope.

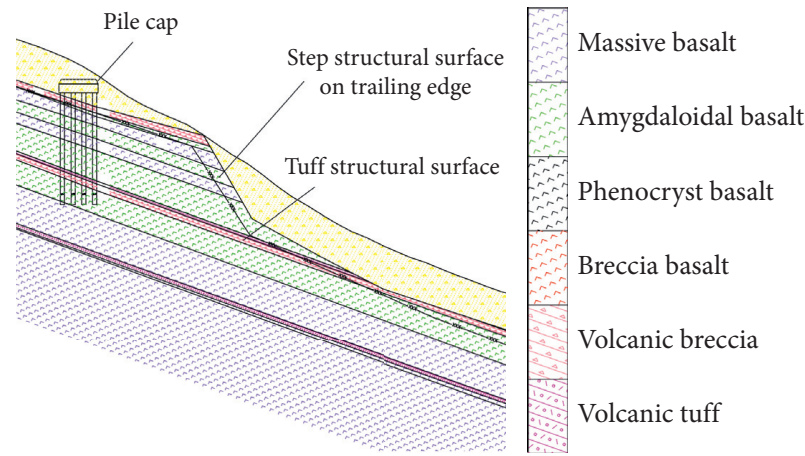

(a)

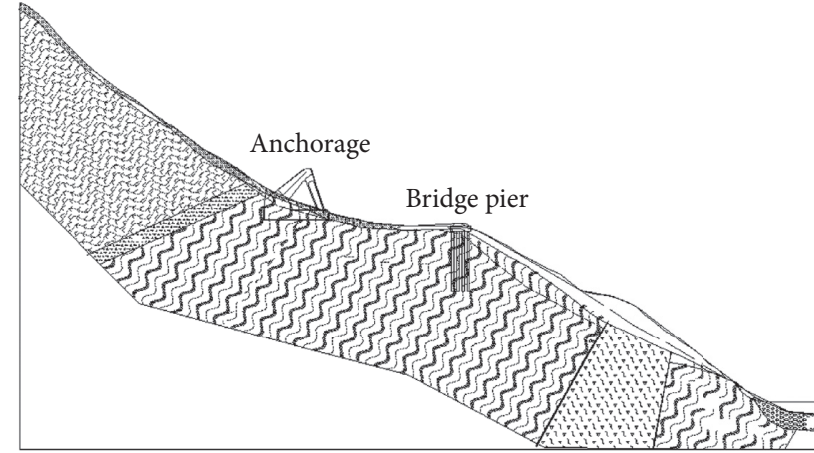

অछs Moderate weathered slate

एक्र Moderate weathered schistosity basalt

Win. Fault fracture zone

(b)

FIgURE 3: Drawing of the slope: (a) Huaping slope; (b) Lijiang slope.

the dynamic response of the slope. The PGA under horizontal seismic load is much larger than that under vertical seismic load. For example, the $\mathrm{PGA}_{\text {max }}$ under horizontal and vertical seismic loads are $1.475 \mathrm{~m} / \mathrm{s}^{2}$ and $1.114 \mathrm{~m} / \mathrm{s}^{2}$, respectively. It indicates that the amplification coefficient under horizontal seismic load is approximately 1.33 times as that under the vertical seismic load, and the amplification effect under the action of horizontal seismic force is greater than that under the vertical seismic force.
Therefore, the P-waves and S-waves mainly induced the dynamic deformation of the slope with discontinuous structural planes, and the dynamic deformation mechanism of the slope can be summarized as follows: first, the slope was shaken by the $\mathrm{P}$-wave, resulting in the formation of many vertical cracks between the discontinuous joints and consequently the formation of the blocks. Then, the slope was shaken by S-wave, and the inertial force of the surface slope becomes larger than that during the $\mathrm{P}$-wave shaking, 


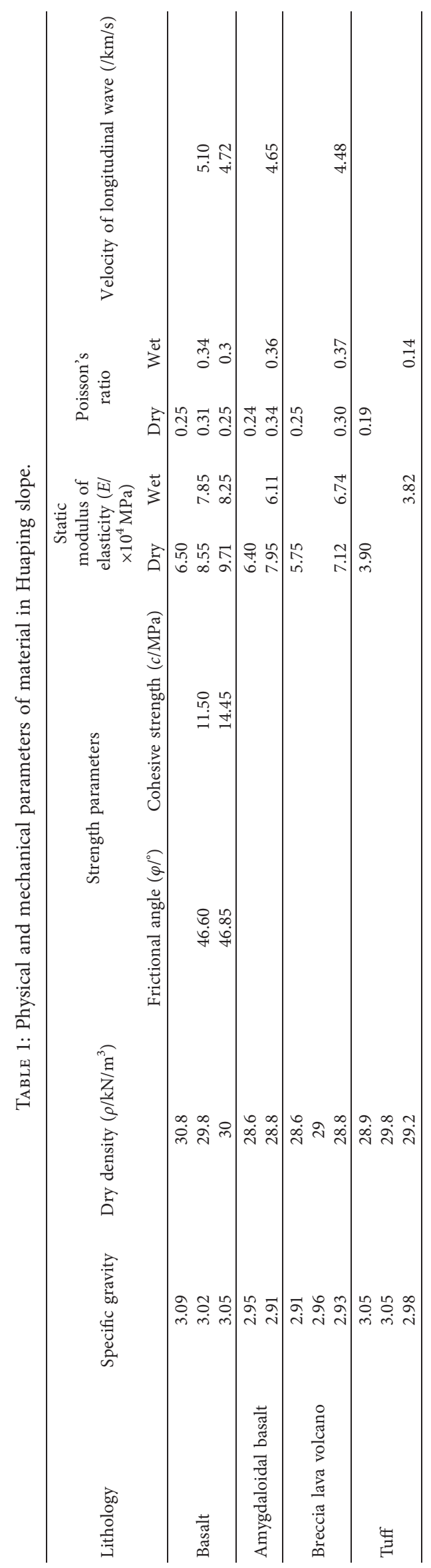




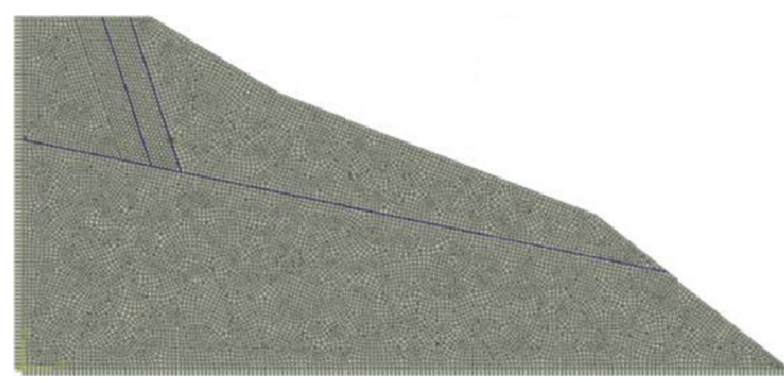

Rock mass element Structural surface element

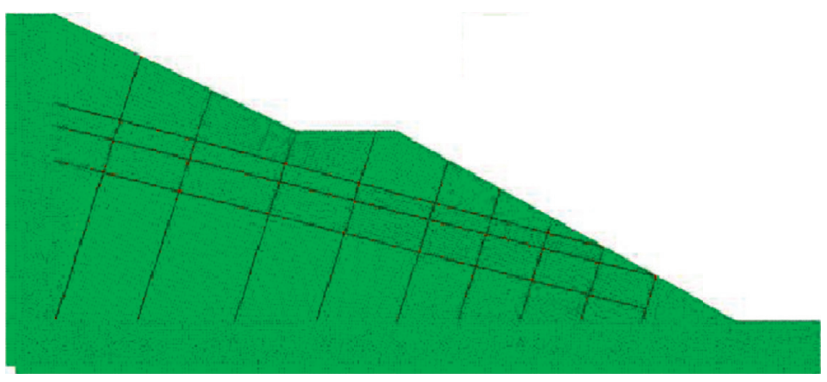

Rock mass element Structural surface element

(a)

(b)

FIgure 4: Mesh model used in the calculations: (a) Huaping slope; (b) Lijiang slope.

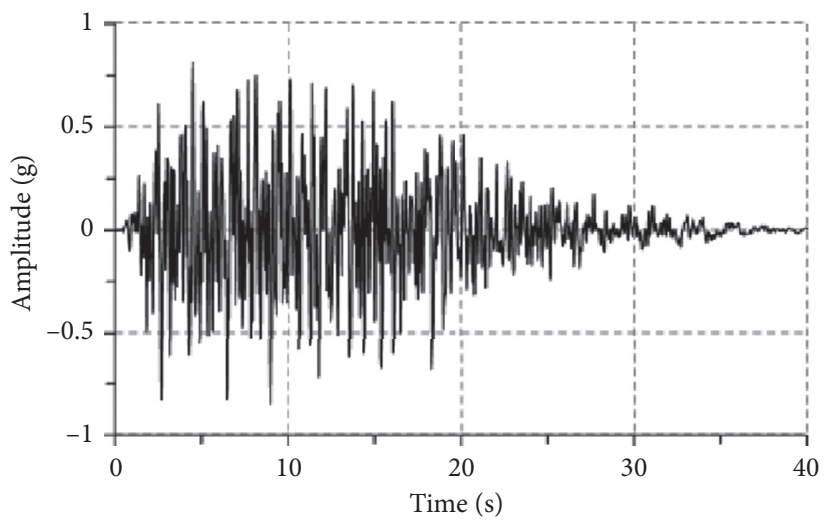

(a)

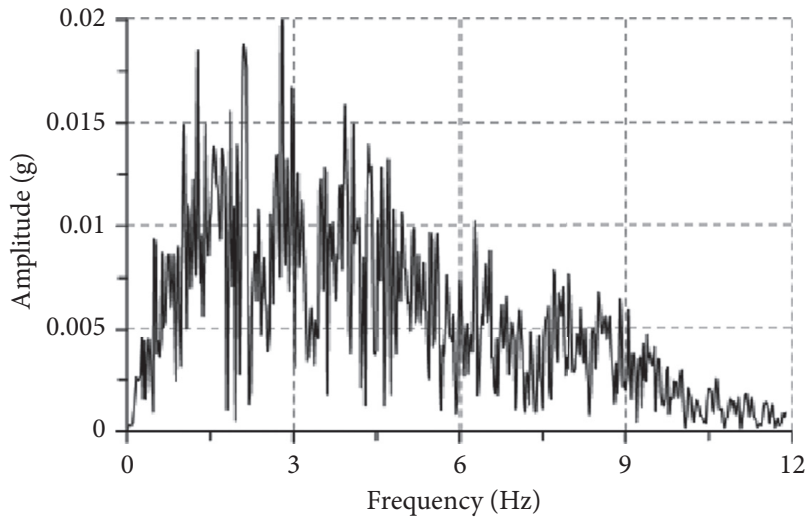

(b)

FIGURE 5: The input seismic wave: (a) time history; (b) Fourier spectrum.

resulting in an obvious magnification of acceleration than that of P-wave. The magnified acceleration effect of the slope was more substantial when the seismic wave was input in the $x$ direction, indicating that the slope deformation was triggered by the S-wave $[7,8,12]$.

\section{Shaking Table Test}

4.1. Shaking Table and Model Container. In this shaking table test, a large-scale electric servo shaking table made by Japan Kokusai Corporation in the Key Laboratory of Loess Earthquake Engineering, Gansu Earthquake Administration, was used. The loading direction contains horizontal direction and vertical direction. The maximum horizontal bearing capacity is $25 \mathrm{t}$, the maximum vertical bearing capacity is $15 \mathrm{t}$, the maximum horizontal acceleration is $1.7 \mathrm{~g}$, and the maximum vertical acceleration is $1.2 \mathrm{~g}$.

A rigid container made of organic glass and carbon steel plates was developed for the tests (Figure 8(b)). Compared to the shear model box, the rigid container has the advantages of light weight, simple structure, and convenient observation of the slope model. The inside dimension of the box (Huaping slope) is $2.8 \mathrm{~m} \times 1.4 \mathrm{~m} \times 1.0 \mathrm{~m}$, while that of the box (Lijiang slope) is $2.8 \mathrm{~m} \times 1.4 \mathrm{~m} \times 1.4 \mathrm{~m}$. In order to reduce the influence of the rigid boundary in the model box, a $200 \mathrm{~mm}$ thick filling layer is set at the end of the model box with loose fine sand in $X$ direction. At the bottom of the box, a $100 \mathrm{~mm}$ thick integral cushion is set with the same material as the slope model.

4.2. Slope Model Construction. The model profile of the Huaping slope and Lijiang slope is shown in Figure 9. The similarity ratio of the model is calculated according to the similarity criterion and Buckingham's $\pi$ theorem of similarity, in combination with the in situ conditions around Jinsha River Bridge. Mechanical properties of the natural materials are listed in Table 1. In the tests, the model geometry $(L)$, mass density $(\rho)$, and seismic acceleration $(a)$ were regarded as the basic dimension, with scaling $C_{L}=375.000, C \rho=1.000$, and $C a=1.000$, respectively. The calculated similarity constant of geometry is 375 , and those of acceleration, strain, and density are all equal to 1 . Other physical quantities, such as density, elastic modulus, cohesion, and internal friction angle of the model material, should be also determined on the basis of similarity principle. Calculated results show that the simulated material of rock mass should have a density of $20.4 \mathrm{kN} / \mathrm{m}^{3}$, elastic 

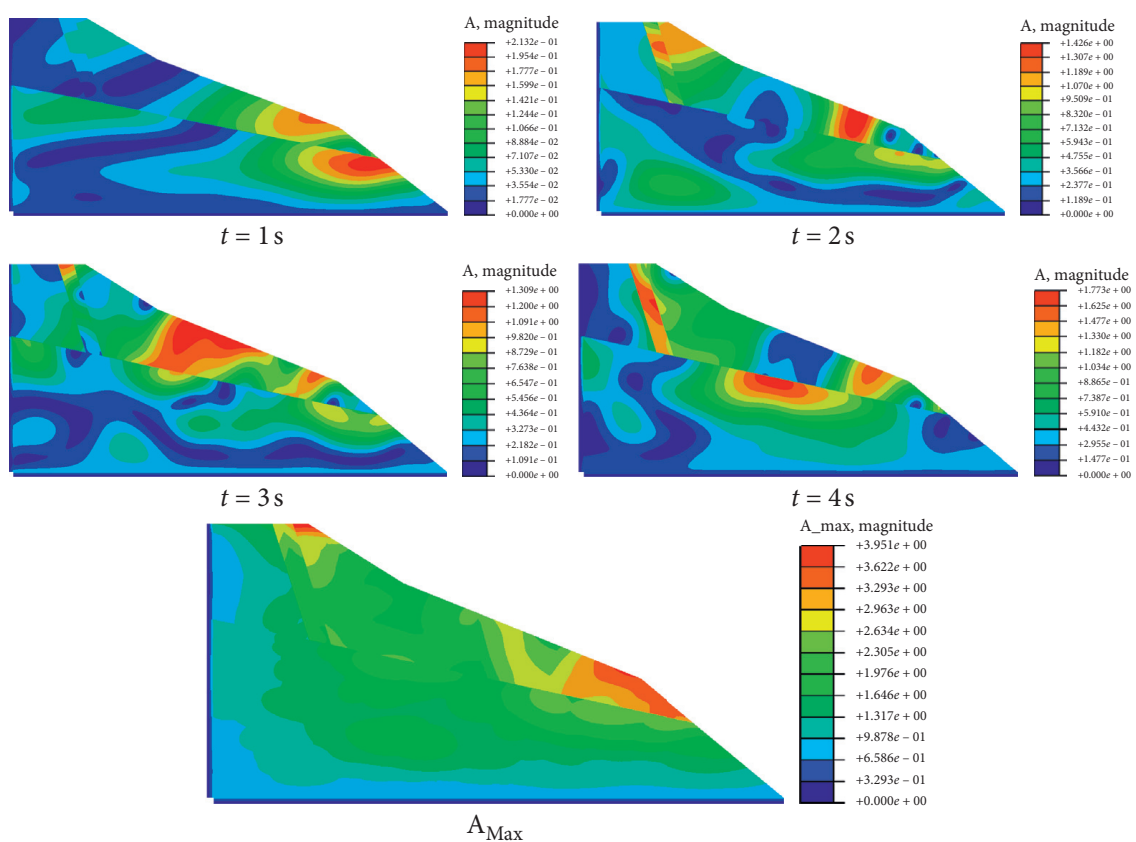

(a)
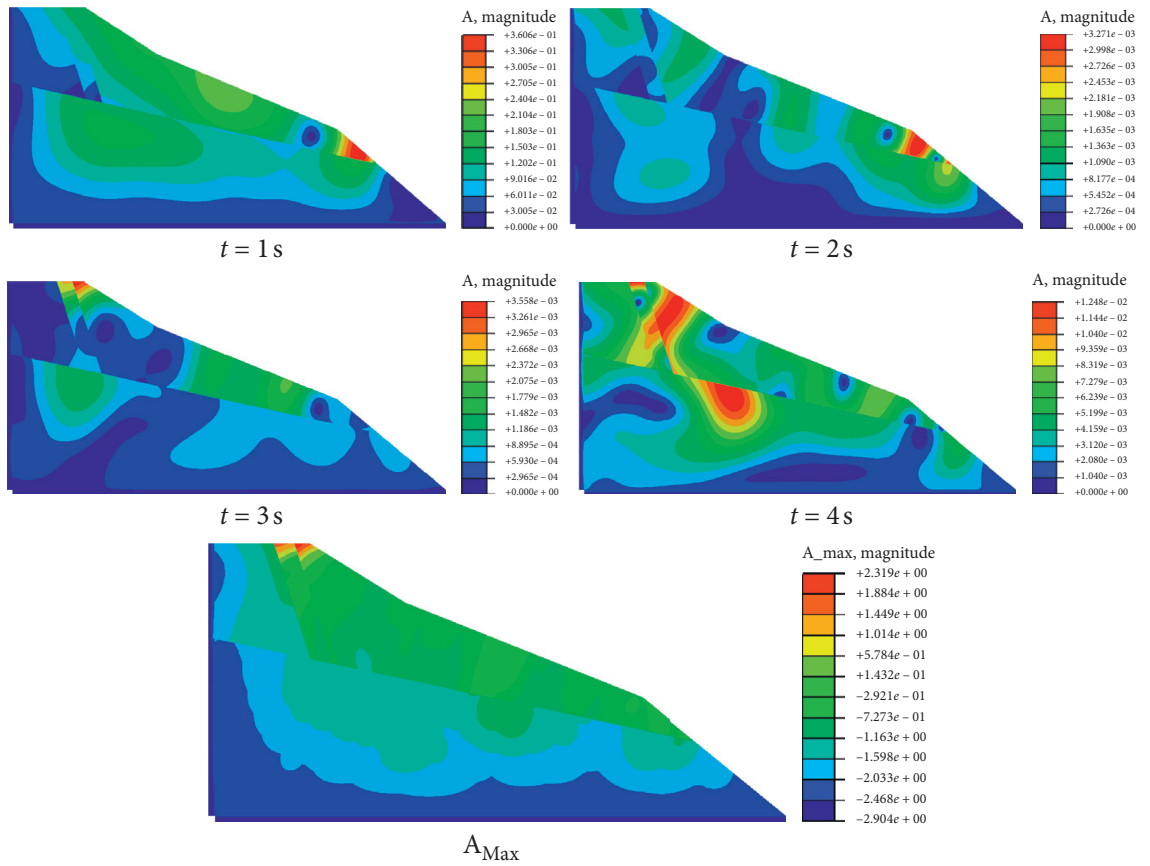

(b)

FIgURE 6: Acceleration response of Huaping slope: (a) input in $x$ direction; (b) input in $z$ direction.

modulus of $100 \mathrm{MPa}$, cohesion of $48.43 \mathrm{kPa}$, and internal friction angle of $40.69^{\circ}$. In order to meet these requirements, the model material is prepared by means of the mixture of other materials, i.e., cement, sand, clay, and iron powder. Through the ratios test, density test, materials test, and static triaxial tests, the mass ratio of cement, sand, clay, iron powder, and mixture (admixture and water reducing agent, water) is chosen as $0.325: 17: 9: 0.4: 0.03: 5$. Therefore, the thickness of the T2 tuff structure surface can be taken as
$5 \mathrm{~mm}$ in the test model. The T2 tuff material on the structure surface is modeled by chevron board to obtain the similar cohesion of $21.1 \mathrm{kPa}$ and internal friction angle of $23.2^{\circ}$ according to the similarity ratio.

The scaled slope model with inclined angle of $60^{\circ}$ is $1.0 \mathrm{~m}$ tall, $1.4 \mathrm{~m}$ wide, and $2.8 \mathrm{~m}$ in length. Teflon tapes were used to simulate the discontinuities and strips of $8 \mathrm{~cm}$-long Teflon tape were spaced $10 \mathrm{~cm}$ apart at $45^{\circ}$ angle to simulate discontinuous joints in the model. The trailing edge of steep 

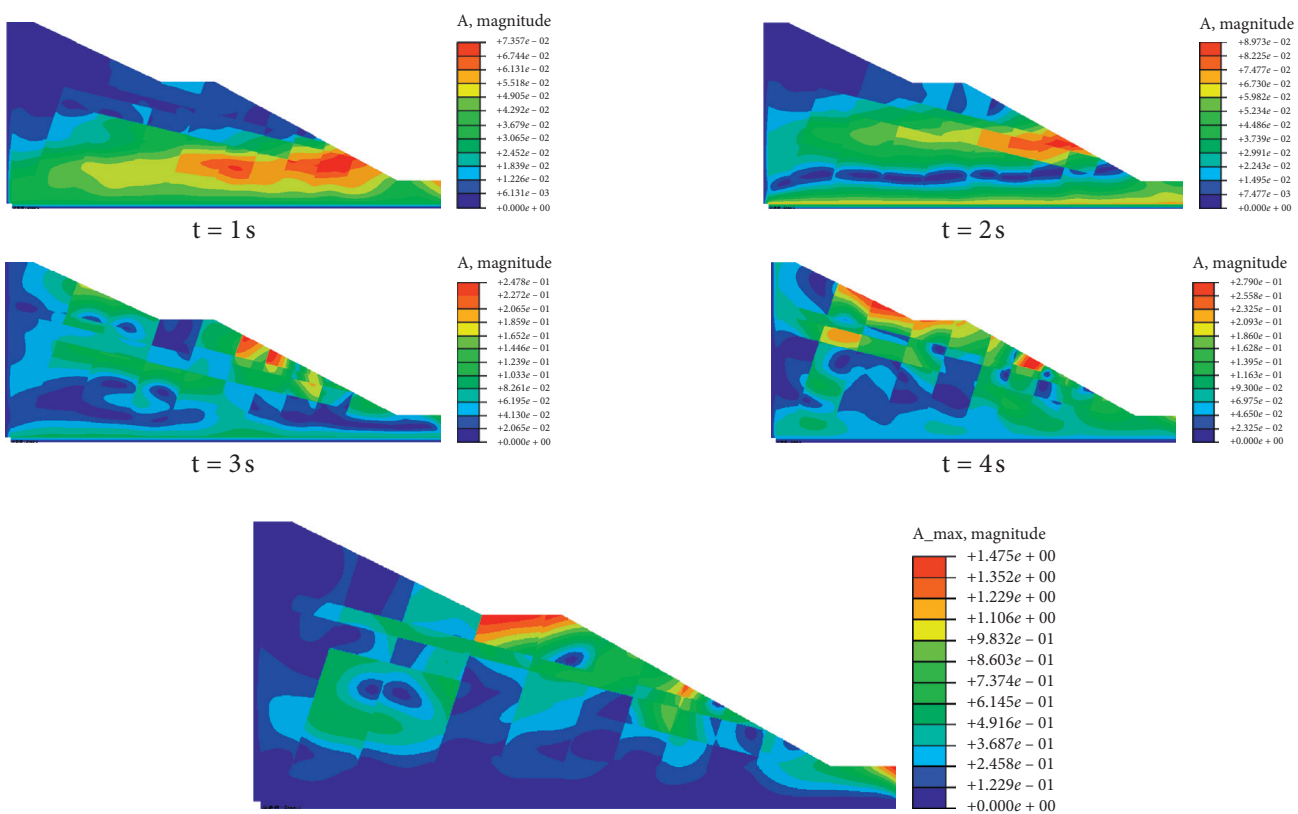

$\mathrm{A}_{\mathrm{Max}}$
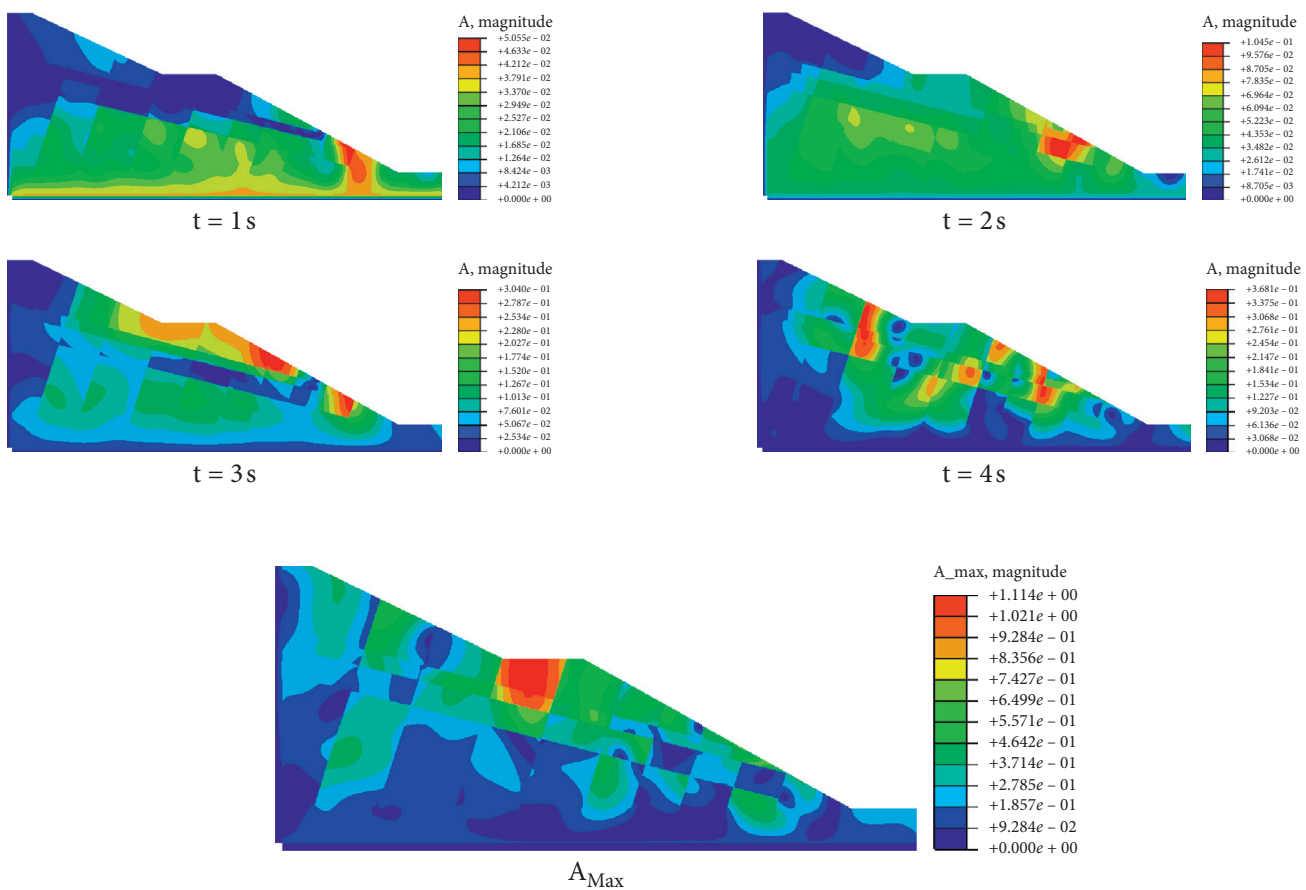

(b)

Figure 7: Acceleration response of Lijiang slope: (a) input in $x$ direction; (b) input in $z$ direction. 


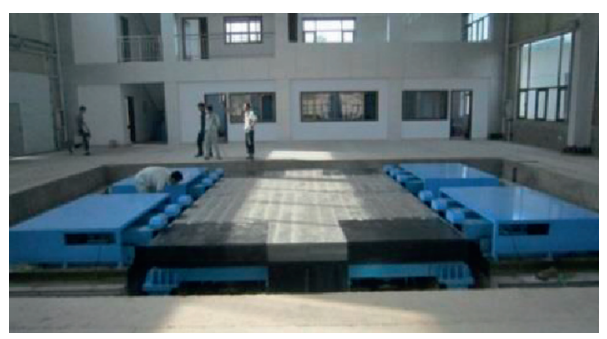

(a)

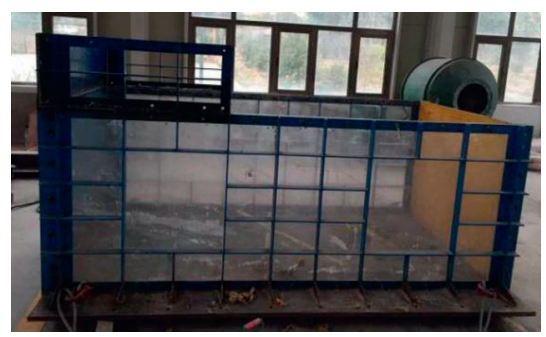

(b)

Figure 8: (a) Large-scale shaking table; (b) model box.

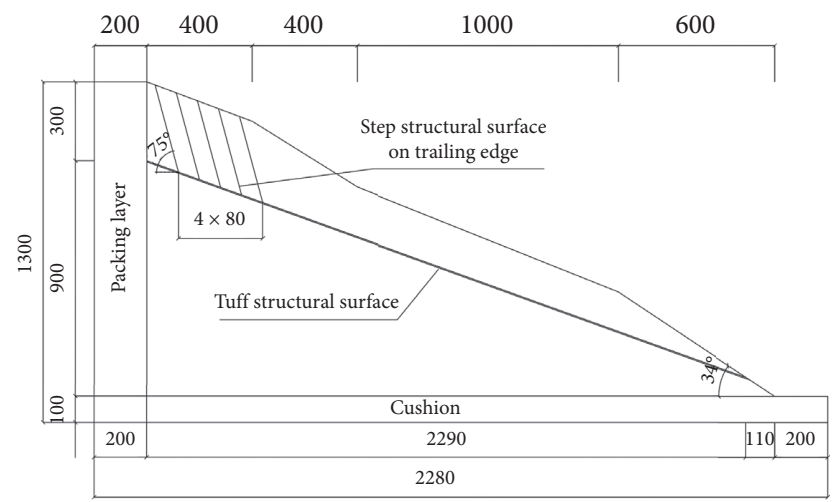

(a)

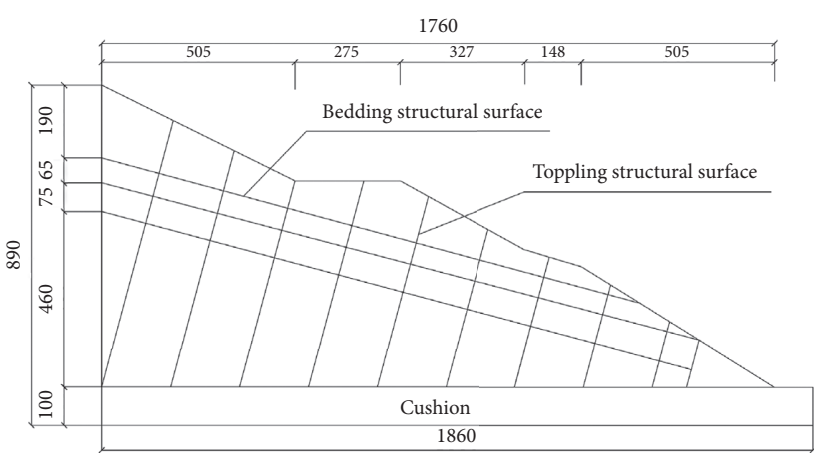

(b)

FIgURE 9: Design of the slope model (unit: mm): (a) Huaping slope; (b) Lijiang slope.

structure surface is simulated by sand seam approach. The slope models were made using the block placement method. The rock mass was split into many blocks made of mixed material, in order to shorten the curing time. Each block was poured with a corresponding mold, and the quality of mixed material was controlled based on the density and volume to ensure the uniformity of slope model. The curing time of blocks was kept the same to guarantee the same strength of mixed material. The blocks are staggered in $Y$ direction to avoid continuous seam. The Huaping slope and Lijiang slope models are shown in Figure 10.

4.3. Loading Cases. The seismic wave was simulated by inputting acceleration time history curve in the process of shaking table tests. The time history and the Fourier spectrum of wave are shown in Figure 11. The Wenchuan earthquake records in $\mathrm{E}-\mathrm{W}$ direction, recorded by Wudu in Gansu of China, were adopted in the test. The dominant frequency of Wenchuan earthquake wave is $7.74 \mathrm{~Hz}$. Two simultaneous loading directions of experimental earthquake excitation (the $z$ and $x$ directions) were applied in the test. The loading sequences of the tests are shown in Tables 2 and 3. Signals recorded from the accelerometers were subjected to undesired noise interferences. It was necessary to perform an appropriate data transformation before the data could be readily used for the subsequent analyses. Prefiltering, zeromean normalization, error removal techniques, and data transformation were applied. The bandwidth of the frequency was set between $3 \mathrm{~Hz}$ and $50 \mathrm{~Hz}$ in the prefiltering process to smooth the response, control the frequencies, and reduce signal noises (particularly high frequency noise from electrical signals) in the data. Blurring in the signals caused by external disturbances or instrument inconsistency was eliminated by the removal of errors.

The measurement points are shown in Figure 12. Twenty accelerometers were installed along two measurement directions. A 3-direction capacitive acceleration sensor (DH301) made by Huadong Testing Co., Ltd., was used, as shown in Figure 10. The sensitivity of this sensor is $-66 \mathrm{mV} /$ $\mathrm{ms}^{2}$ and its error is $\pm 20 \mathrm{~m} / \mathrm{s}^{2}$. Additionally, its frequency range is $0-1500 \mathrm{~Hz}$ horizontally and $0-800 \mathrm{~Hz}$ vertically.

\subsection{Dynamic Response of Acceleration}

4.4.1. Influence of Structural Surface on the Amplification Effect. Figure 13 shows that the distribution of the acceleration amplification coefficient of the measurement points in Huaping slope. It was found that the acceleration was clearly amplified along the slope surface and reached maximum at the top of the slope. The PGA amplification coefficient increases with the increase of seismic intensity gradually, under the horizontal and vertical seismic loads. This phenomenon indicates that the seismic intensity has a great impact on the dynamic response of the slope.

To investigate the dynamic acceleration response of the Lijiang slope with discontinuous structural surfaces, taking 


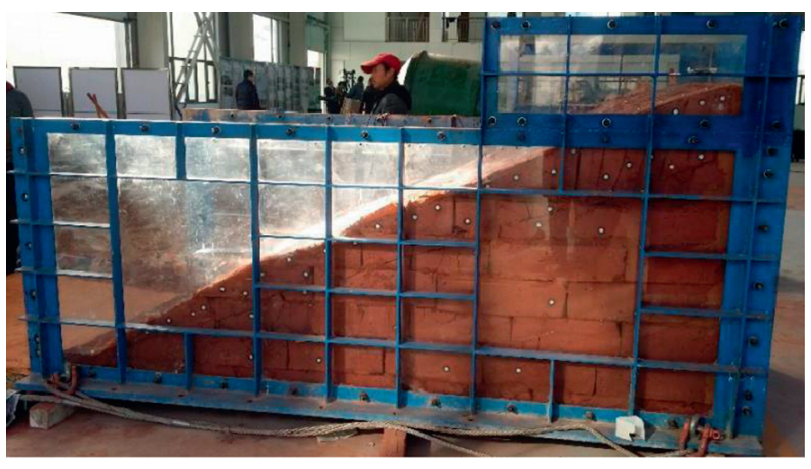

(a)

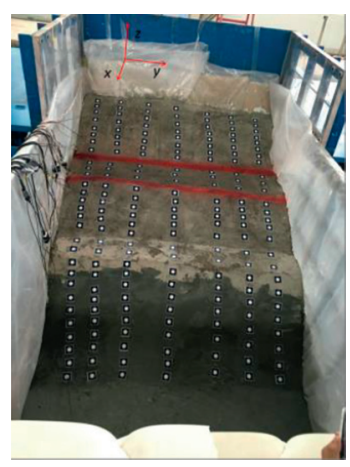

(b)

FIGURE 10: Soil container and the scaled model: (a) Huaping slope; (b) Lijiang slope.

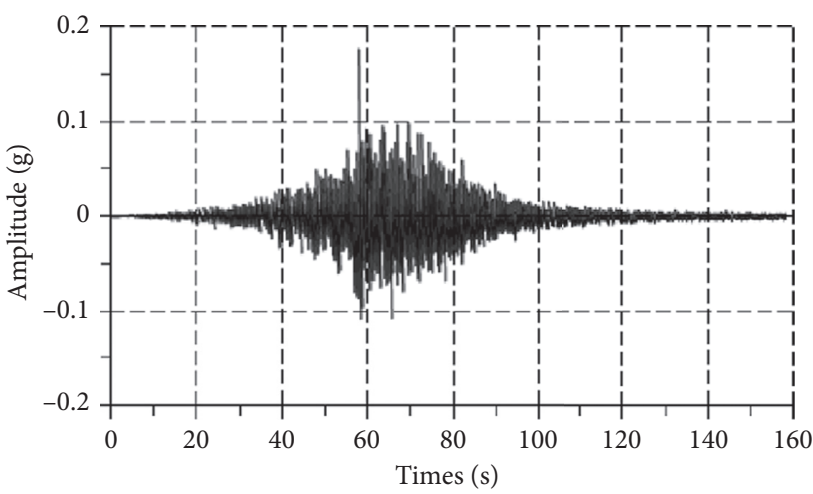

(a)

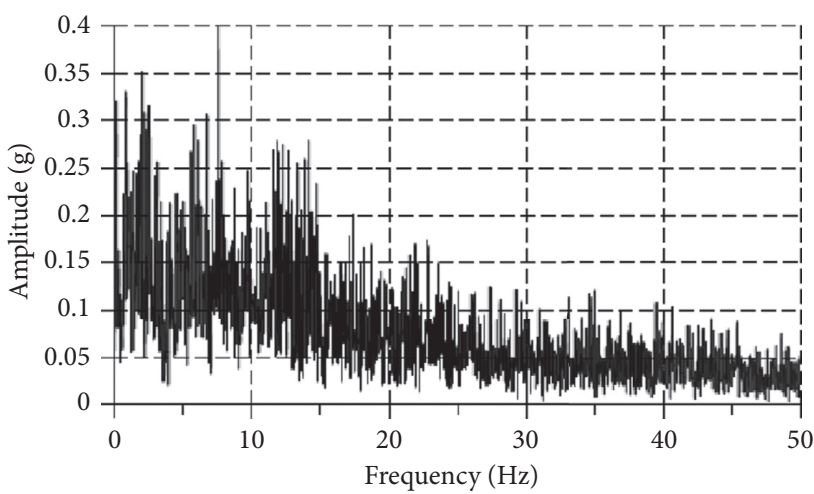

(b)

FIgURE 11: The input Wenchuan earthquake wave data: (a) time history; (b) Fourier spectrum.

TABLE 2: Loading sequence of Huaping slope.

\begin{tabular}{lcccccccc}
\hline Amplitude (g) & 0.037 & 0.074 & 0.037 & 0.074 & 0.297 & 0.446 & 0.297 & 0.446 \\
\hline No & 1 & 2 & 3 & 4 & 5 & 6 & 7 & 8 \\
Vibration direction & $z$ & $z$ & $x$ & $x$ & $z$ & $z$ & $x$ \\
\hline
\end{tabular}

TABLE 3: Loading sequence of Lijiang slope.

\begin{tabular}{lcccccccc}
\hline Amplitude (g) & 0.084 & 0.084 & 0.168 & 0.168 & 0.336 & 0.336 & 0.504 & 0.504 \\
\hline No & 1 & 2 & 3 & 4 & 5 & 6 & 7 & 8 \\
Vibration direction & $z$ & $x$ & $z$ & $x$ & $z$ & $x$ & $z$ & $x$ \\
\hline
\end{tabular}

$0.084 \mathrm{~g}$ and $0.336 \mathrm{~g}$ as examples, the distribution of $\mathrm{M}_{\mathrm{PGA}}$ under vertical and horizontal seismic loads is shown in Figure 14. Figure 14 shows that the amplification effect in the upper layer of slope is much larger than that in the internal slope. It can be also found that the $\mathrm{M}_{\mathrm{PGA}}$ has a certain increase with the seismic intensity increasing. On the whole, the increasing rate $\mathrm{M}_{\mathrm{PGA}}$ changes differently with the change of earthquake intensity, and the change trend is not linear but shows obviously nonlinear increasing trend. This phenomenon indicates that the acceleration amplification effect is different under different seismic loads and also suggests that the dynamic deformation is also different under different seismic intensities.
4.4.2. Influence of Seismic Wave Direction of the Amplification Effect. Taking Lijiang bank slope as an example, the acceleration amplification coefficient $\left(\mathrm{M}_{\mathrm{PGA}}\right)$ inside and on the slope surface under horizontal and vertical seismic forces is shown in Figure 15. As can be seen from Figure 15, under the action of vertical seismic force and horizontal seismic force, the $\mathrm{M}_{\mathrm{PGA}}$ of the internal slope and slope surface increases with the increase of elevation gradually and reach the maximum value at the top of slope. It is worth noting that the $\mathrm{M}_{\mathrm{PGA}}$ under the action of vertical seismic force is less than that under the action of horizontal seismic force, and it indicates that the acceleration amplification effect 


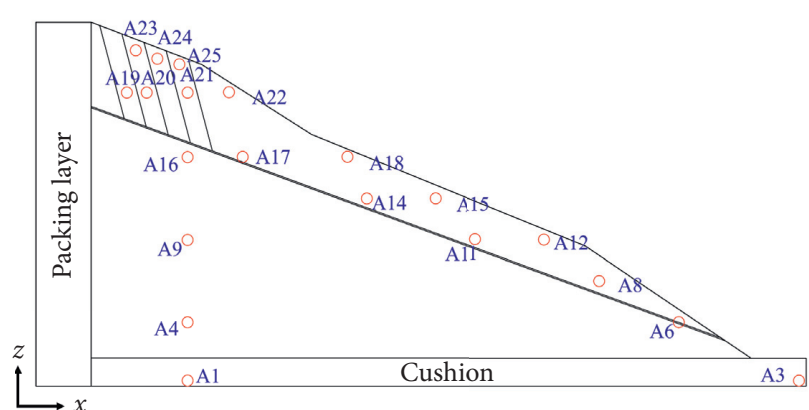

- Sensor location

A Acceleration sensor

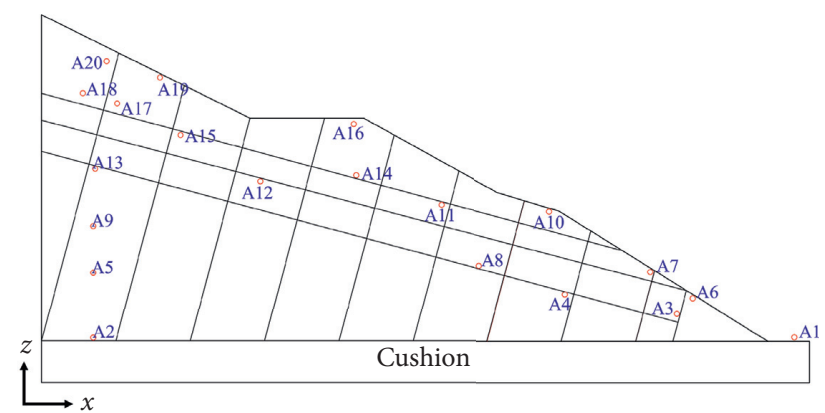

- Sensor location

A Acceleration sensor

(a)

(b)

FIgURE 12: Acceleration sensor distribution (unit: mm): (a) Huaping slope; (b) Lijiang slope.
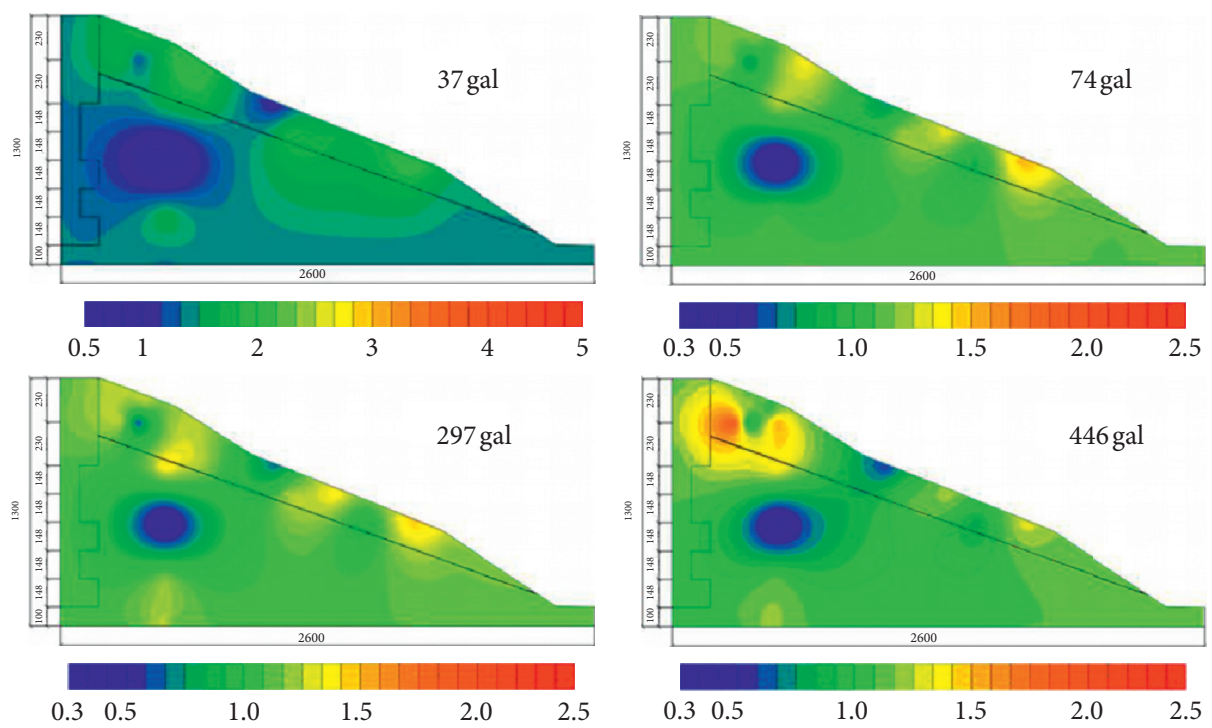

(a)
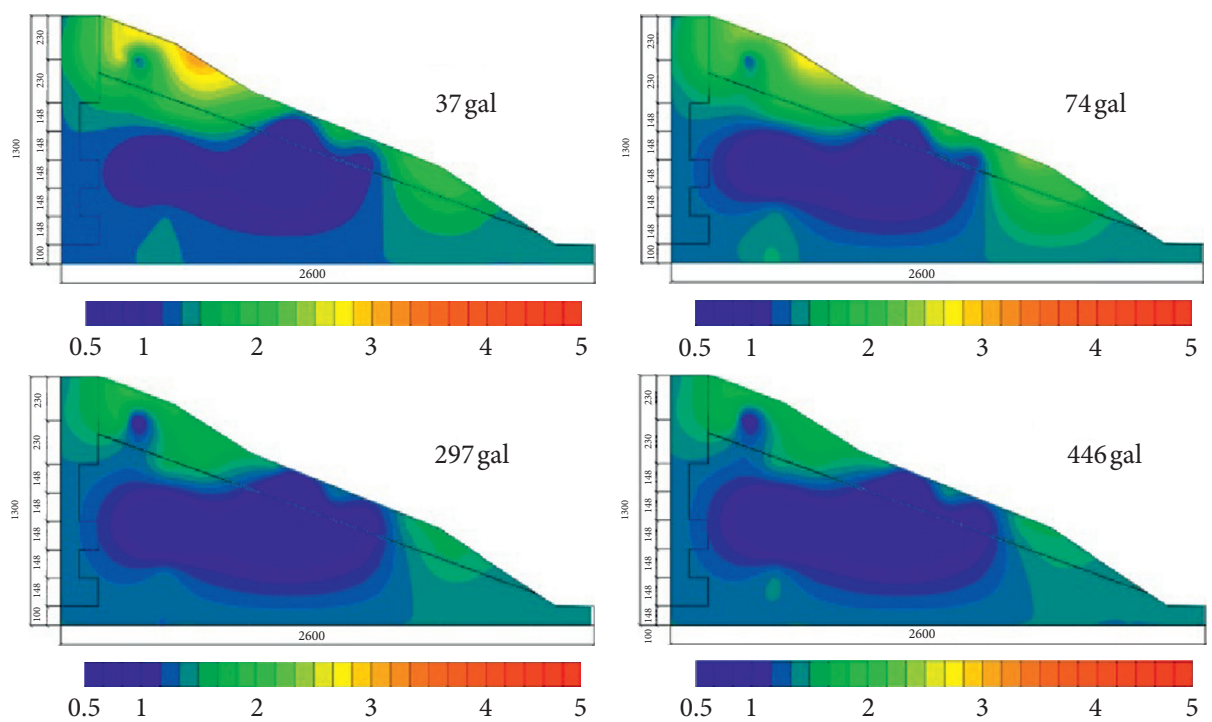

(b)

Figure 13: Acceleration amplification coefficient distribution of the Huaping slope model: (a) input in $z$ direction; (b) input in $x$ direction. 


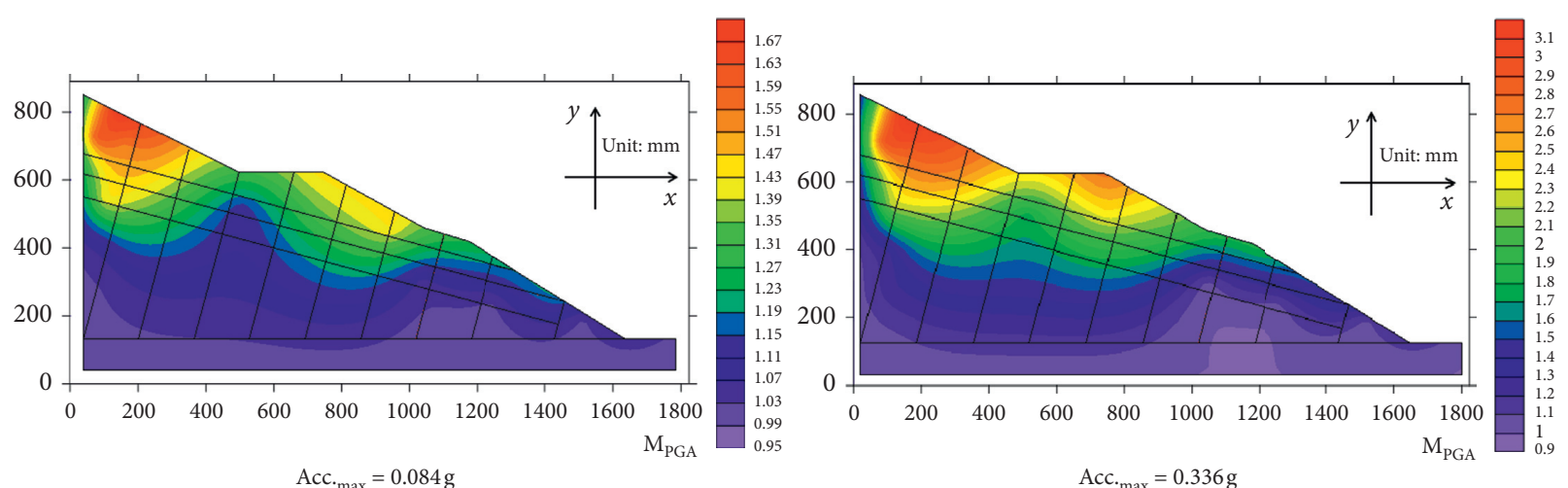

(a)
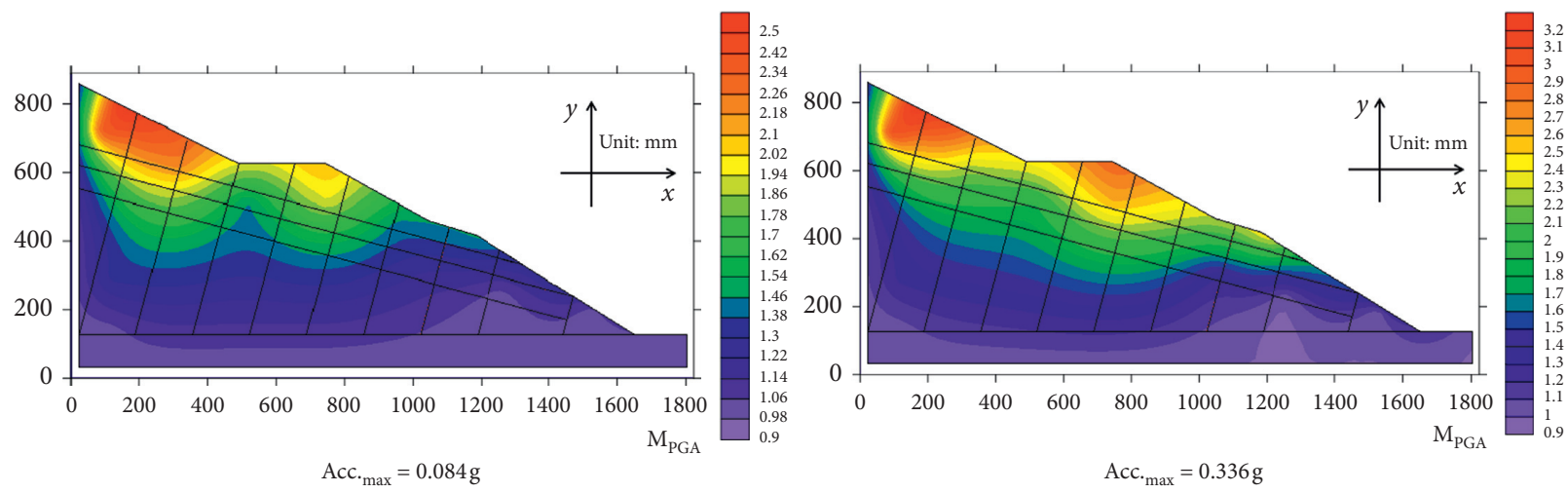

(b)

FIgURE 14: Acceleration amplification coefficient distribution of the Lijiang slope model: (a) input in $z$ direction; (b) input in $x$ direction.

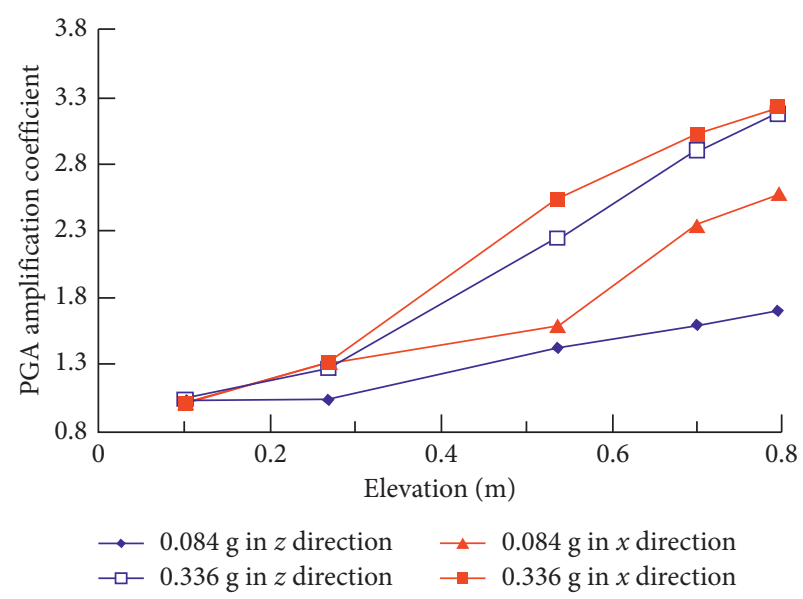

(a)

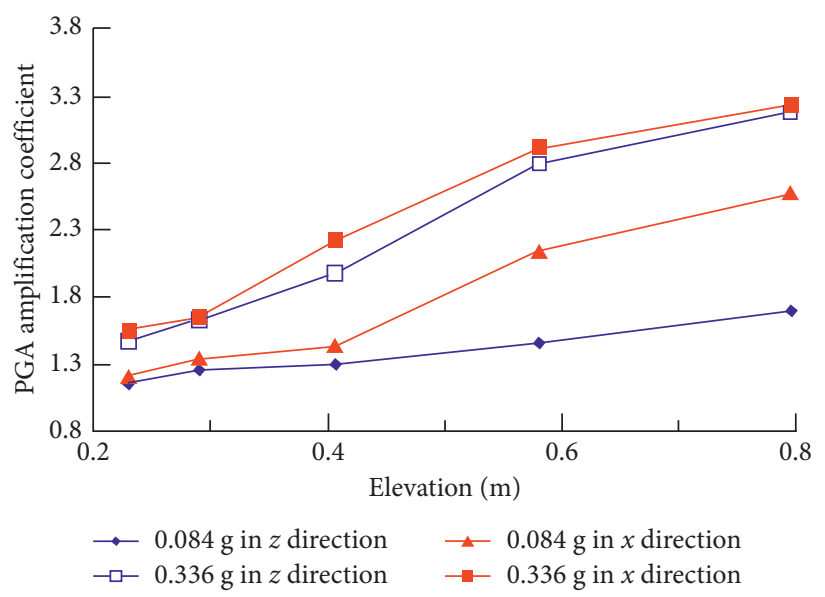

(b)

Figure 15: Acceleration amplification coefficient change rule of Lijiang slope: (a) inside the slope; (b) near the slope surface.

under the action of horizontal seismic force is greater than that under the action of vertical seismic force, which is consistent with the results of numerical simulation. In particular, the maximum acceleration amplification coefficient $\left(\mathrm{M}_{\mathrm{PGAmax}}\right)$ under the vertical seismic force at the top of the slope is similar to that under the horizontal seismic force. When the input motion is intense like $0.336 \mathrm{~g}$, the cracks around the structural surface developed and the blocks segmented by structural surfaces on slope crest became loose parts, resulting in the similar amplification effect in different directions.

Compared with the slope of Huaping bank (Figure 13), the deformation of the break of Huaping bank is mainly controlled by the bedding structural surface. As can be seen from Figure 13, the amplification effect of acceleration under the action of vertical seismic force is weak. Given the 


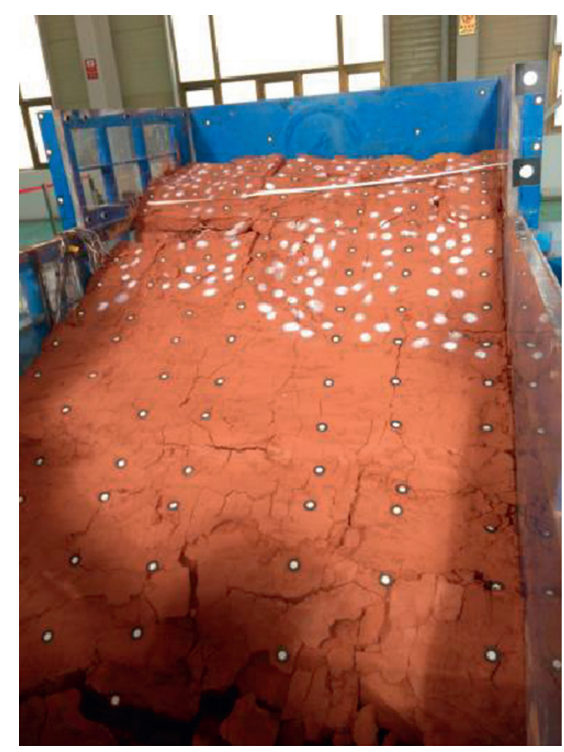

(a)

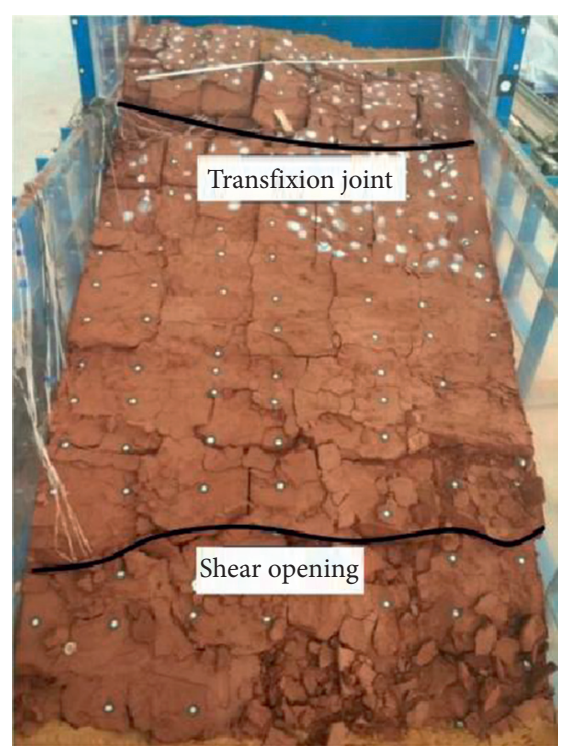

(b)
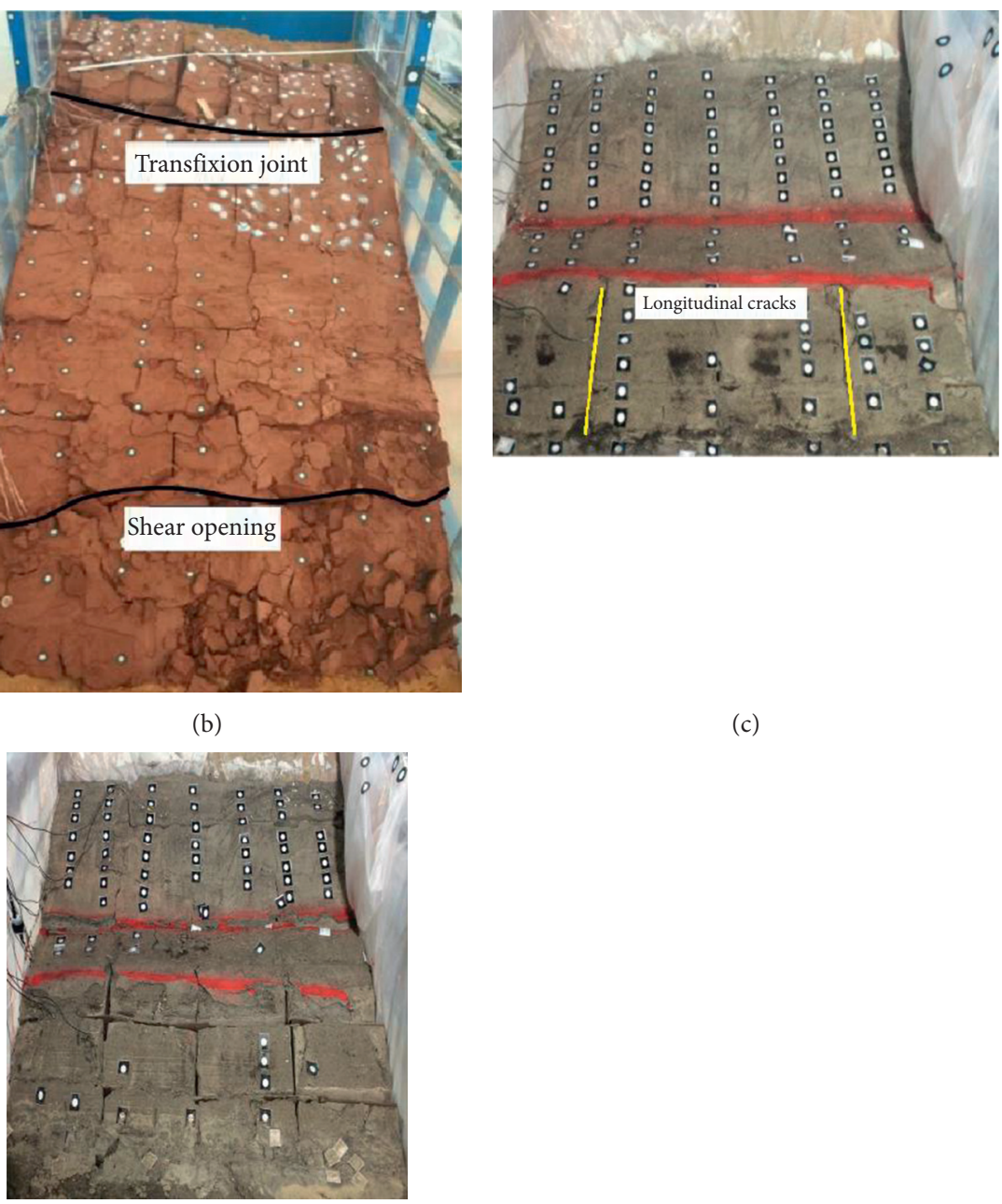

(c)

(d)

FIGURE 16: The damage phenomenon of the slope: (a) the damage phenomenon of Huaping slope under vertical seismic load; (b) the damage phenomenon of Huaping slope under horizontal seismic load; (c) the damage phenomenon of Lijiang slope under vertical seismic load; (d) the damage phenomenon of Lijiang slope under horizontal seismic load.

abovementioned analysis, the amplification effect of vertical seismic force on Huaping bank slope is weak, while that of Lijiang bank slope is obvious. However, the destruction effect of horizontal seismic force still dominates. Therefore, the input wave directions have a great impact on the acceleration effect of the slope; particularly, the input wave in $x$ direction has a great effect on the amplification effect of the slope.

In the shaking table test, the results of acceleration amplification coefficient and numerical simulation are consistent in distribution trend, but there are some differences in magnitude. The main reason is that the model in the numerical simulation is constructed according to the actual size of slope, while the model in the shaking table test is reduced according to the similarity criterion. Although the similarity criterion considers the similarity between the geometric and physical conditions of the model, it is difficult to fully simulate the nonlinear mechanical behavior of rock mass. So, there are some differences between numerical simulation and the shaking table test in results of amplification coefficient of the slope model. This analysis result is consistent with the field investigation in Wenchuan earthquake. The earthquake-induced rock landslides are characterized by rough and serrate fracture surfaces, according to field investigations in the damaged area. Most of the landslides near the epicenter of the Wenchuan earthquake were subjected to strong lateral S-wave forces and were characterized by "throw-like collapses." Therefore, seismic force can "throw out" the surface plant without little change of original geomorphology.

4.5. Failure Mechanism. Huang et al. [24] proposed that the failure mechanism in various rock slopes was similar. The Huaping slope and Lijiang slope are the slopes with weak structural planes, and their failure mechanisms own similar rule. From the observations, it can be concluded that the deformations induced by the earthquake mainly occurred at the upper part of the slope. Numerous fresh fractures were formed in the rock mass along the discontinuity joints, resulting in cataclysmic structures. 


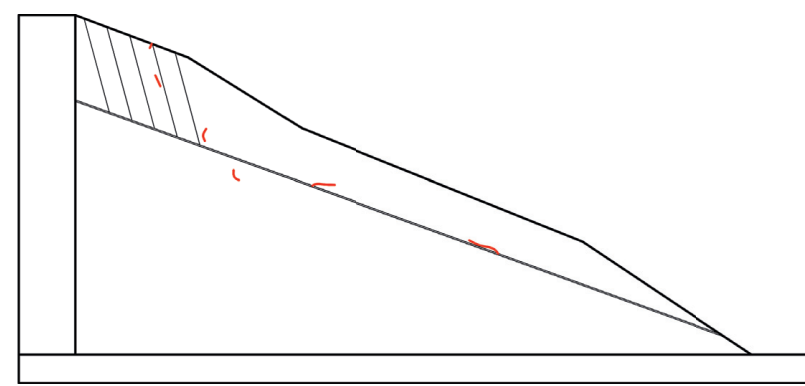

(a)

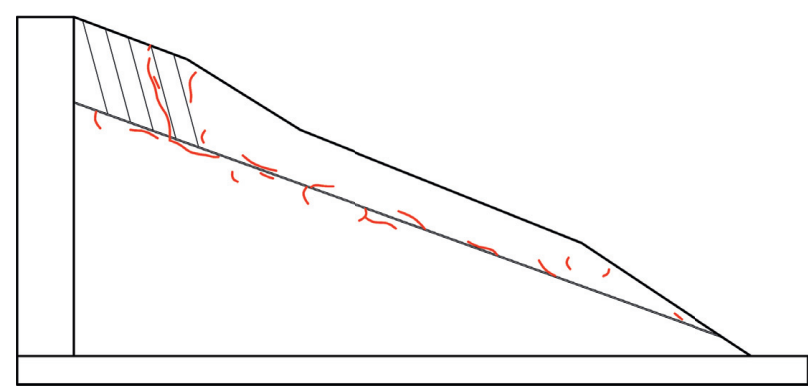

(c)

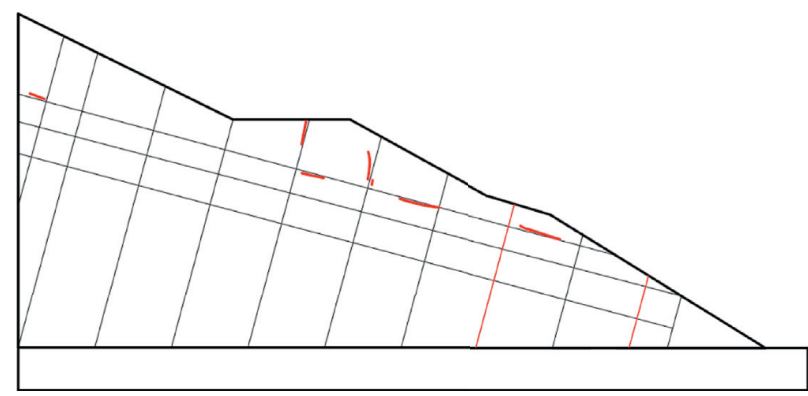

(e)

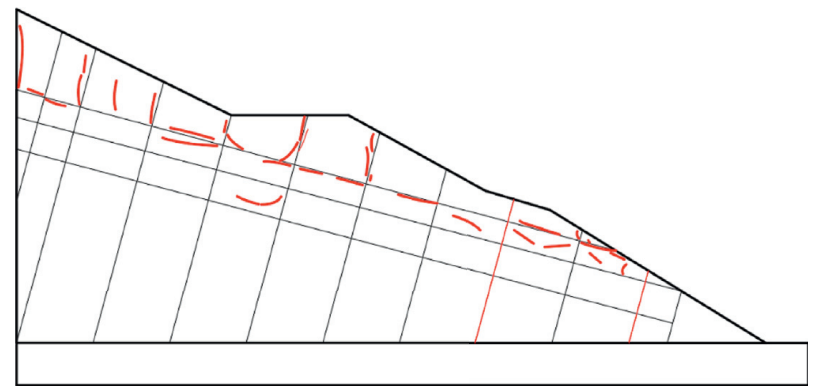

(g)

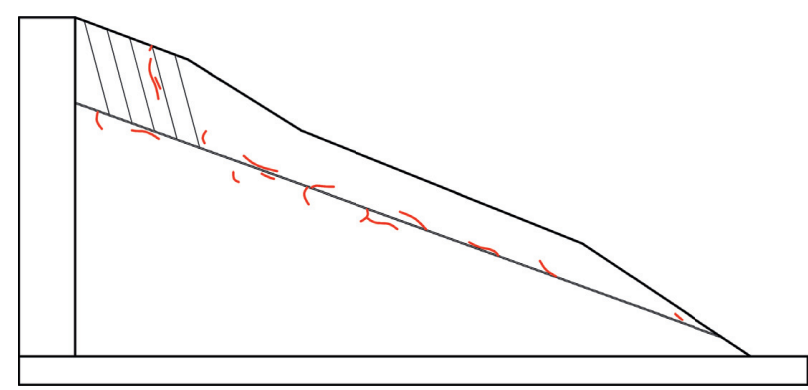

(b)

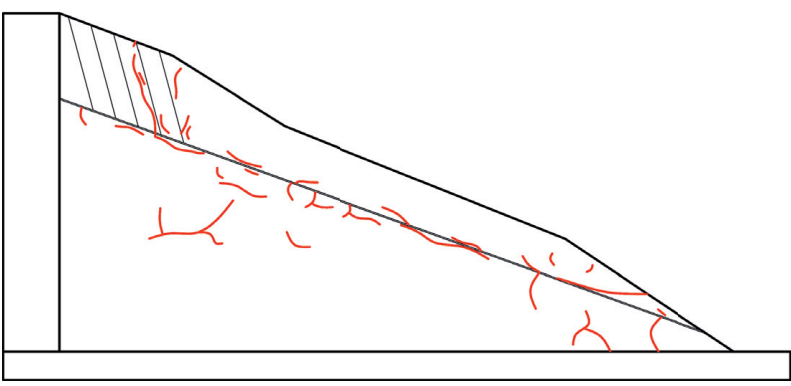

(d)

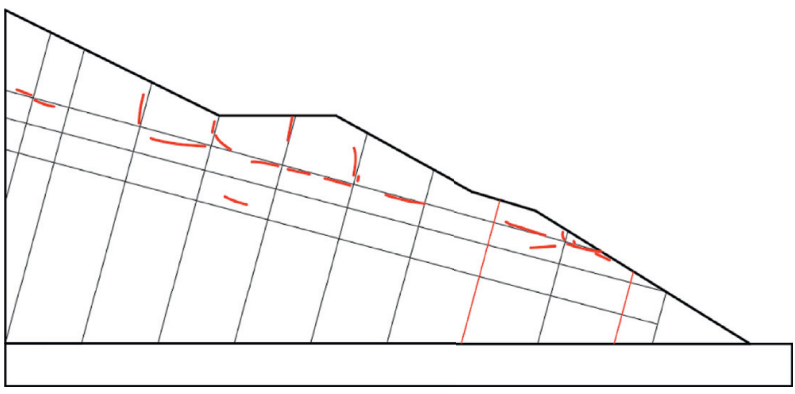

(f)

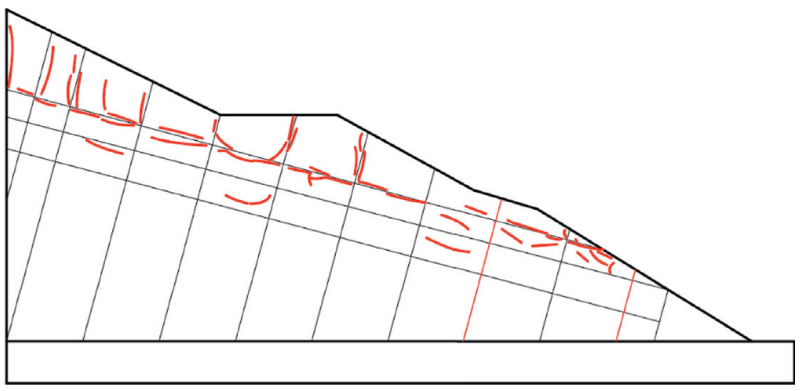

(h)

FIGURE 17: The dynamic damage process of the slopes: (a) Huaping slope (0.074 g in $z$ direction); (b) Huaping slope (0.074 g in $x$ direction); (c) Huaping slope $\left(0.297 \mathrm{~m} / \mathrm{s}^{2}\right.$ in $z$ direction); (d) Huaping slope (0.446 g in $x$ direction); (e) Lijiang slope (0.168 g in $z$ direction); (f) Lijiang slope (0.168 $\mathrm{g}$ in $x$ direction); (g) Lijiang slope (0.504 $\mathrm{g}$ in $z$ direction); (h) Lijiang slope (0.504 $\mathrm{g}$ in $x$ direction).

In addition, Figures 16(a) and 16(b), the failure deformation of the Huaping bank slope model under the action of vertical seismic force is smaller than that under the action of horizontal seismic force. Figures 16(c) and 16(d) show that the deformation degree of the Lijiang bank slope model under the action of horizontal seismic force is also greater than that under the action of vertical seismic force. Figure 17 illustrates the dynamic damage process of two slope models. The failure phenomenon and damage process also verify the above analysis results of the acceleration amplification effect.
The amplification effect of horizontal seismic force is larger than that of vertical seismic force. The slope surface of Huaping slope shows overall shear opening failure under earthquake action, as shown in Figure 16(b). Horizontal seismic motion plays a major control role. And it is worth noting that, in Lijiang bank slope, it makes the slope under the action of vertical seismic force surface longitudinal cracks in the tilting structure, slope surface of the block has uneven settlement, the effect of vertical seismic force in Lijiang bank has the main effect on the failure mode of the 
slope, due to that the Lijiang bank both forward and reverse bend structure surface, and the slope belongs to block structure. This is because blocks of slope are loose under the action of vertical seismic motion, and the horizontal seismic motion makes the block produce reciprocating shear motion until the failure of slope. The horizontal and vertical seismic motions control the slope failure together, and the vertical seismic motion cannot be ignored in the slope stability evaluation. When the deformation is controlled by the forward structural surface, the horizontal seismic force station plays a leading role, while the vertical force has a small impact. In the case of forward and reverse control, due to the formation of block structure, the vertical force has a greater impact on the vertical crack and a greater impact on the deformation than the former.

\section{Conclusion}

The wave propagation characteristics of the two model slopes with bedding structural surface and discontinuous structural surfaces were assessed by the dynamic FEM analyses and the large-scaled shaking table test. Some conclusions can be made as follows:

For the rock slopes with structural surfaces, the acceleration amplification effect increases with the slope elevation. The slope surface is the main amplification area during earthquakes. In particular, compared with the Huaping slope, the Lijiang slope shows irregular wave propagation characteristics. All these results reveal the great effect of structural surfaces on the acceleration amplification effect. The refraction and reflection phenomenon can be found during the wave propagation through the structural plane. The seismic wave directions also have impact on the dynamic response of the slope. Compared with the vertical seismic load, the amplification effect of the slope is much larger under horizontal seismic excitation.

A series of shaking table tests was carried out to study the dynamic stability of the rock slopes. The acceleration response of the rock slope mainly developed within a shallow depth zone in the upper part of the slope. It is observed that the top area of the slope will completely be collapsed along a slide surface once the input acceleration amplitude reaches a certain value. In addition, the location of the sliding plane in the model is consistent with the location of the maximum horizontal acceleration. A large deformation at the top of the slope will be generated under seismic loading. The results show that the seismic wave directions have impact on the dynamic response of the slope. Compared with the vertical seismic load, the amplification effect of the slope is much larger. The amplification coefficient of seismic acceleration at the top of Huaping slope under horizontal seismic excitation is about twice of that under vertical seismic excitation. The amplification coefficients of seismic acceleration at the top of Lijiang slope under horizontal and vertical seismic excitation are 3.23 and 3.18 , respectively.

\section{Data Availability}

The data used to support the findings of this study are available from the corresponding author upon request.

\section{Conflicts of Interest}

The authors declare that they have no conflicts of interest.

\section{Acknowledgments}

This work was supported by the Science and Technology Project of Department of Transport of Yunnan Province (2018 [45]) and the Science and Technology Demonstration Project of the Ministry of Transport (2017-09).

\section{References}

[1] T. Kamai and A. Sangawa, "Landslides on ancient embankments in the kinki district (Japan): strong motion seismoscope of the 1596 keichou-fushimi earthquake," Quaternary International, vol. 242, no. 1, pp. 90-105, 2011.

[2] T. Miyagi, D. Higaki, H. Yagi et al., "Reconnaissance report on landslide disasters in northeast Japan following the M 9 Tōhoku earthquake," Landslides, vol. 8, no. 3, pp. 339-342, 2011.

[3] R. Huang and W. Li, "Development and distribution of geohazards triggered by the 5.12 wenchuan earthquake in China," Science in China Series E: Technological Sciences, vol. 52, no. 4, pp. 810-819, 2009.

[4] R. Q. Huang and W. L. Li, "Analysis of the geo-hazards triggered by the 12 may 2008 wenchuan earthquake, China," Bulletin of Engineering Geology and the Environment, vol. 68, no. 3, pp. 363-371, 2009.

[5] H. B. Havenith and C. Bourdeau, "Earthquake-induced hazards in mountain regions: a review of case histories from Central Asia-an inaugural lecture to the society," Geologica Belgica, vol. 13, pp. 135-150, 2010.

[6] F. C. Dai, C. Xu, X. Yao, L. Xu, X. B. Tu, and Q. M. Gong, "Spatial distribution of landslides triggered by the $2008 \mathrm{~ms} 8.0$ wenchuan earthquake, China," Journal of Asian Earth Sciences, vol. 40, no. 4, pp. 883-895, 2011.

[7] D. Song, A. Che, R. Zhu, and X. Ge, "Dynamic response characteristics of a rock slope with discontinuous joints under the combined action of earthquakes and rapid water drawdown," Landslides, vol. 15, no. 6, pp. 1109-1125, 2018.

[8] D. Song, A. Che, Z. Chen, and X. Ge, "Seismic stability of a rock slope with discontinuities under rapid water drawdown and earthquakes in large-scale shaking table tests," Engineering Geology, vol. 245, pp. 153-168, 2018.

[9] Y. Huang, W. Zhang, Q. Xu, P. Xie, and L. Hao, "Run-out analysis of flow-like landslides triggered by the ms 8.02008 wenchuan earthquake using smoothed particle hydrodynamics," Landslides, vol. 9, no. 2, pp. 275-283, 2012.

[10] R. Huang, X. Pei, X. Fan, W. Zhang, S. Li, and B. Li, "The characteristics and failure mechanism of the largest landslide triggered by the wenchuan earthquake, may 12, 2008, China," Landslides, vol. 9, no. 1, pp. 131-142, 2012.

[11] X. P. Li and S. M. He, "Seismically induced slope instabilities and the corresponding treatments: the case of a road in the Wenchuan Earthquake hit region," Journal of Mountain Science, vol. 6, no. 1, pp. 90-100, 2009.

[12] A. L. Che, H. K. Yang, B. Wang, and X. R. Ge, "Wave propagations through jointed rock masses and their effects on the stability of slopes," Engineering Geology, vol. 201, no. 9, pp. 45-56, 2015.

[13] D. Song, X. Feng, Z. Wang, and H. Song, "Using near-real-time monitoring of landslide deformation to interpret hydrological 
triggers in jiudian gorge reservoir," Indian Journal of Geo-Marine Sciences, vol. 46, no. 11, pp. 2182-2190, 2017.

[14] D. Song, S. Liang, and Z. Wang, "The influence of reservoir filling on a preexisting bank landslide stability," Indian Journal of Geo-Marine Sciences, vol. 47, no. 2, pp. 291-300, 2018.

[15] H. H. Li, C. H. Lin, W. Zu, C. C. Chen, and M. C. Weng, "Dynamic response of a dip slope with multi-slip planes revealed by shaking table tests," Landslides, vol. 15, pp. 1731-1743, 2018.

[16] M. Jiang, T. Jiang, G. B. Crosta, Z. Shi, H. Chen, and N. Zhang, "Modeling failure of jointed rock slope with two main joint sets using a novel dem bond contact model," Engineering Geology, vol. 193, pp. 79-96, 2015.

[17] D. Song, J. Chen, and J. Cai, "Deformation monitoring of rock slope with weak bedding structural plane subject to tunnel excavation," Arabian Journal of Geosciences, vol. 11, no. 11, p. 251, 2018.

[18] J.-J. Wang, H.-P. Zhang, L. Zhang, and Y. Liang, "Experimental study on heterogeneous slope responses to drawdown," Engineering Geology, vol. 147-148, no. 5, pp. 52-56, 2012.

[19] G. Fan, J. Zhang, J. Wu, and K. Yan, "Dynamic response and dynamic failure mode of a weak intercalated rock slope using a shaking table," Rock Mechanics and Rock Engineering, vol. 49, no. 8, pp. 1-14, 2016.

[20] Y. S. Hong, R. H. Chen, C. S. Wu, and J. R. Chen, "Shaking table tests and stability analysis of steep nailed slopes," $\mathrm{Ca}$ nadian Geotechnical Journal, vol. 42, no. 5, pp. 1264-1279, 2011.

[21] V. S. Gischig, E. Eberhardt, J. R. Moore, and O. Hungr, "On the seismic response of deep-seated rock slope instabilitiesinsights from numerical modeling," Engineering Geology, vol. 193, pp. 1-18, 2015.

[22] Z.-L. Chen, X. Hu, and Q. Xu, "Experimental study of motion characteristics of rock slopes with weak intercalation under seismic excitation," Journal of Mountain Science, vol. 13, no. 3, pp. 546-556, 2016.

[23] Y. Huang and X. Jiang, "Field-observed phenomena of seismic liquefaction and subsidence during the 2008 wenchuan earthquake in China," Natural Hazards, vol. 54, no. 3, pp. 839-850, 2010.

[24] R. Huang, J. Zhao, N. Ju, G. Li, M. L. Lee, and Y. Li, “Analysis of an anti-dip landslide triggered by the 2008 wenchuan earthquake in China," Natural Hazards, vol. 68, no. 2, pp. 1021-1039, 2013.

[25] H. Liu, Q. Xu, Y. Li, and X. Fan, "Response of high-strength rock slope to seismic waves in a shaking table test," Bulletin of the Seismological Society of America, vol. 103, no. 6, pp. 3012-3025, 2013.

[26] H.-X. Liu, Q. Xu, and Y.-R. Li, "Effect of lithology and structure on seismic response of steep slope in a shaking table test," Journal of Mountain Science, vol. 11, no. 2, pp. 371-383, 2014.

[27] B. Sreenivas, B. Kumar, and B. K. Raghu Prasad, "Investigation of the ductility demand in multi-story buildings subjected to near field ground motions using neural network approach," Journal of Earthquake Engineering, vol. 12, no. 8, pp. 1314-1324, 2008.

[28] A. Aziminejad and A. S. Moghadam, "Fragility-based performance evaluation of asymmetric single-story buildings in near field and far field earthquakes," Journal of Earthquake Engineering, vol. 14, no. 6, pp. 789-816, 2010.
[29] M. Raghunandan and A. B. Liel, "Effect of ground motion duration on earthquake-induced structural collapse," Structural Safety, vol. 41, no. 3, pp. 119-133, 2013.

[30] Z. Zembaty, A. Castellani, and G. Boffi, "Spectral analysis of the rotational component of seismic ground motion," Probabilistic Engineering Mechanics, vol. 8, no. 1, pp. 5-14, 1993.

[31] A. P. Young, P. N. Adams, W. C. O’Reilly, R. E. Flick, and R. T. Guza, "Coastal cliff ground motions from local ocean swell and infragravity waves in southern California," Journal of Geophysical Research Oceans, vol. 116, no. C9, 2015.

[32] C. Smerzini, R. Paolucci, and M. Stupazzini, "Experimental and numerical results on earthquake-induced rotational ground motions," Journal of Earthquake Engineering, vol. 13, no. sup1, pp. 66-82, 2009.

[33] M.-L. Lin and K.-L. Wang, "Seismic slope behavior in a largescale shaking table model test," Engineering Geology, vol. 86, no. 2-3, pp. 118-133, 2006.

[34] G. M. Latha and A. Garaga, "Seismic stability analysis of a himalayan rock slope," Rock Mechanics and Rock Engineering, vol. 43, no. 6, pp. 831-843, 2010.

[35] C. Xu, X. Xu, X. Yao, and F. Dai, "Three (nearly) complete inventories of landslides triggered by the may 12, 2008 wenchuan mw 7.9 earthquake of China and their spatial distribution statistical analysis," Landslides, vol. 11, no. 3, pp. 441-461, 2014.

[36] B. Xu and C. Yan, "An experimental study of the mechanical behavior of a weak intercalated layer," Rock Mechanics and Rock Engineering, vol. 47, no. 2, pp. 791-798, 2014.

[37] L. P. Wang and G. Zhang, "Centrifuge model test study on pile reinforcement behavior of cohesive soil slopes under earthquake conditions," Landslides, vol. 11, no. 2, pp. 213-223, 2014.

[38] Z. Zhang, T. Wang, S. Wu, H. Tang, and C. Liang, "Seismic performance of loess-mudstone slope in Tianshui-centrifuge model tests and numerical analysis," Engineering Geology, vol. 222, pp. 225-235, 2017.

[39] Z. Sun, L. Kong, A. Guo, G. Xu, and W. Bai, "Experimental and numerical investigations of the seismic response of a rock-soil mixture deposit slope," Environmental Earth Sciences, vol. 78, p. 716, 2019.

[40] K. Yan, J. Zhang, Q. Cheng, J. Wu, Z. Wang, and H. Tian, "Earthquake loading response of a slope with an inclined weak intercalated layer using centrifuge modeling," Bulletin of Engineering Geology and the Environment, vol. 78, no. 6, pp. 4439-4450, 2019. 